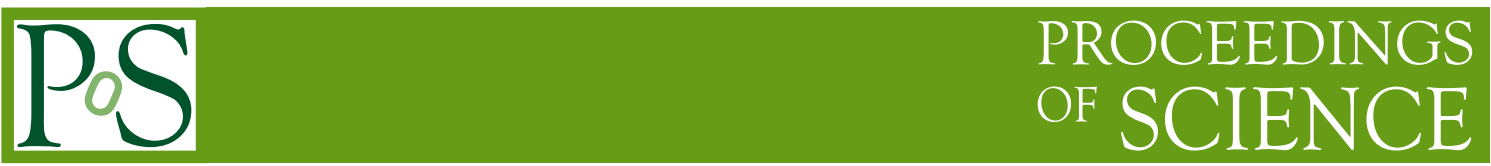

\title{
Continuum limit and universality of the Columbia plot
}

\section{Philippe de Forcrand*}

Institut für Theoretische Physik, ETH Zürich, CH-8093 Zürich, Switzerland

CERN, Theory Division, CH-1211 Geneva 23, Switzerland

E-mail: forcrandephys.ethz.ch

\section{Massimo D’Elia}

INFN - Sezione di Pisa, Largo Pontecorvo 3, I-56127 Pisa, Italy

Dipartimento di Fisica dell'Università di Pisa, Largo Pontecorvo 3, I-56127 Pisa, Italy

E-mail: massimo.delia@unipi.it

\begin{abstract}
Results on the thermal transition of QCD with 3 degenerate flavors, in the lower-left corner of the Columbia plot, are puzzling. The transition is expected to be first-order for massless quarks, and to remain so for a range of quark masses until it turns second-order at a critical quark mass. But this critical quark mass and resulting "pion" mass disagree violently between Wilson and staggered fermions at finite lattice spacing, and decrease sharply with the lattice spacing, for staggered fermions at least. To clarify this puzzle and eliminate potential systematic effects from rooting, we study the 4-flavor theory with staggered fermions, on lattices with 4 to 10 timeslices. Our results are qualitatively similar to the 3-flavor case, so that rooting is not an issue. However, dramatic cutoff effects are visible, even on our finest lattices. Universality implies that cutoff effects for Wilson fermions are even more dramatic. In order to obtain a first-order thermal transition in the continuum theory, extremely light quarks are needed.
\end{abstract}

34th annual International Symposium on Lattice Field Theory

24-30 July 2016

University of Southampton, UK

\footnotetext{
* Speaker.
} 


\section{Introduction}
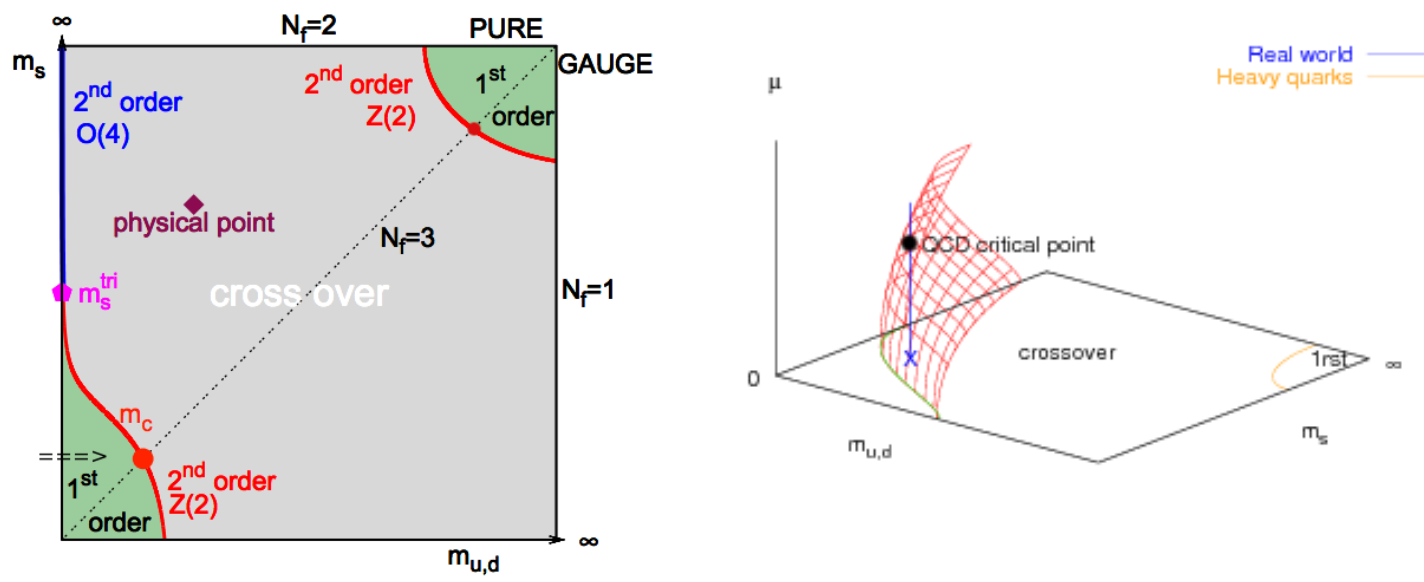

Figure 1: (left) Columbia plot: we focus on the $N_{f}=3$ chiral critical point (arrow), and its $N_{f}=4$ analogue; (right): adding a vertical axis for the chemical potential $\mu$, a possible QCD chiral critical point occurs when the surface swept by the $\mu=0$ chiral critical line intersects the physical quark masses' vertical line.

The QCD phase diagram summarizes the various behaviors of QCD as a function of temperature $T$ and matter density, or equivalently quark chemical potential $\mu$. Since the chiral and the center symmetry, which play crucial roles in the phase diagram, are both explicitly broken in QCD by the quark masses, it is useful to consider these masses as QCD parameters: $m_{u, d}$ for the two light quark masses considered degenerate for simplicity, and $m_{s}$ for the strange quark mass. Our expectations for the $\mu=0$ phase diagram, projected along the $T$-direction, are contained in the "Columbia plot" Fig. 1 (left).

The upper-right and lower-left corners of the Columbia plot are simpler to analyze:

- In the first, all quarks are infinitely massive. They decouple, and the resulting $S U\left(N_{c}=3\right)$ YangMills theory obeys the global $Z(3)$ center symmetry, which is spontaneously broken at high temperature via a first-order transition.

- In the second, all quarks are massless, and the theory obeys the global $S U\left(N_{f}=3\right)$ chiral symmetry, which is spontaneously broken at low temperature and restored at high temperature. For 3 massless flavors or more, one expects symmetry restoration to occur via a first-order transition [1], because no $3 d S U\left(N_{f}\right), N_{f} \geq 3$, second-order universality class is known [2].

In the middle of the Columbia plot, where both symmetries are badly broken explicitly, Monte Carlo simulations indicate an analytic crossover as $T$ is raised. Thus, there must exist two critical, second-order lines separating the two first-order regions above from the central crossover region. Because no particular symmetry is at play along these critical lines, their universality class should be that of a $3 d \phi^{4}$ theory, i.e. that of the $3 d$ Ising model.

A simple way to pin down the location of these two critical lines is to consider the $N_{f}=3$ case, with all quark masses equal, shown as the diagonal of the Columbia plot. Two critical quark masses should be observed, to be determined with high precision via Monte Carlo simulations. In practice, it is difficult to adopt a reference scale, since an $N_{f}=3$ theory is a distortion of real-world 


\begin{tabular}{ccccc} 
Action & $N_{t}$ & $m_{\pi}^{c}$ & Ref. & Year \\
\hline standard staggered & 4 & $\sim 290 \mathrm{MeV}$ & {$[6]$} & 2001 \\
p4 staggered & 4 & $\sim 67 \mathrm{MeV}$ & {$[7]$} & 2004 \\
standard staggered & 6 & $\sim 150 \mathrm{MeV}$ & {$[8]$} & 2007 \\
HISQ staggered & 6 & $\lesssim 45 \mathrm{MeV}$ & {$[9]$} & 2011 \\
stout staggered & $4-6$ & could be zero & {$[10]$} & 2014 \\
\hline Wilson-clover & $6-8$ & $\sim 300 \mathrm{MeV}$ & {$[5]$} & 2014 \\
Wilson-clover & $4-10$ & $\sim 100 \mathrm{MeV}$ & {$[11]$} & 2016 \\
\hline
\end{tabular}

Table 1: Summary of previous studies of the $N_{f}=3, \mu=0$ chiral critical point - adapted from [10]. The general trend is: finer lattices and/or improved actions drive the critical "pion" mass down; Wilson fermions favor much heavier values. The last line (Wilson, $N_{t}=10$ ) was presented at the Lattice conference [11]. Other, related studies have kept fixed to their physical value the strange quark mass [12], or the ratio $m_{s} / m_{u, d}[13]$.

QCD. So, it is convenient to trade the critical quark mass for the ratio of the corresponding $T=0$ "pion" mass $m_{\pi}^{c}$ over the transition temperature $T_{c}: m_{\pi}^{c} / T_{c}$ is of order 1 for real-world QCD, and allows to separate the regime of "light" and that of "heavy" quarks.

Besides increasing our fundamental knowledge, the quantitative determination of the Columbia plot is useful when considering the effect of a chemical potential $\mu$, which can be viewed as an additional vertical axis. The two $\mu=0$ critical lines discussed above will sweep critical surfaces as $\mu$ is turned on. The chiral critical surface, near the lower-left corner, may bend away from the origin, and reach the physical quark mass values for a sufficiently large $\mu$ : this signals the presence of a QCD chiral critical point, as in Fig. 1 (right). Or this critical surface may bend the other way, and there may be no chiral critical point [3]. Even in the first case, reaching the critical point will require more bending if the $\mu=0$ critical line corresponds to smaller quark masses. Thus, an accurate determination of this critical line is an important ingredient to shape our understanding of the finite-density properties of QCD. This can all be studied at $\mu=0$, without having to face the "sign problem" present at non-zero chemical potential.

The importance of pinning down the $\mu=0, N_{f}=3$ critical points has been recognized. The technical difficulty is to control the approach to the continuum limit. For the heavy quark case, the masses are $\gtrsim \mathscr{O}(1) \mathrm{GeV}$, which requires fine lattices to avoid UV cutoff effects. Numerical work so far has focused on coarse lattices with $N_{t}=4$ time-slices [4]. Such difficulties are absent in the light quark case, so that one would expect reasonable accuracy for lattice spacings $\mathscr{O}(0.1)$ fm, i.e. $N_{t} \gtrsim 8$. However, one observes $(i)$ large cutoff effects $(\sim 30 \%)$ for $N_{t}=4,6,8$ [5], and (ii) enormous discrepancies (a factor of $\sim$ four!) between staggered and Wilson fermions at these values of $N_{t}$.

Table I, adapted from [10], summarizes the results of past studies, and adds some results presented at the Lattice conference [11]. The general trend is: the more one approaches the continuum limit, by decreasing the lattice spacing or by improving the action, the softer the transition, and the lighter the critical "pion" is. The most remarkable case is that of [10]: using a stout-improved action with staggered fermions on an $N_{t}=4$ lattice, no sign of a first-order transition was found, 


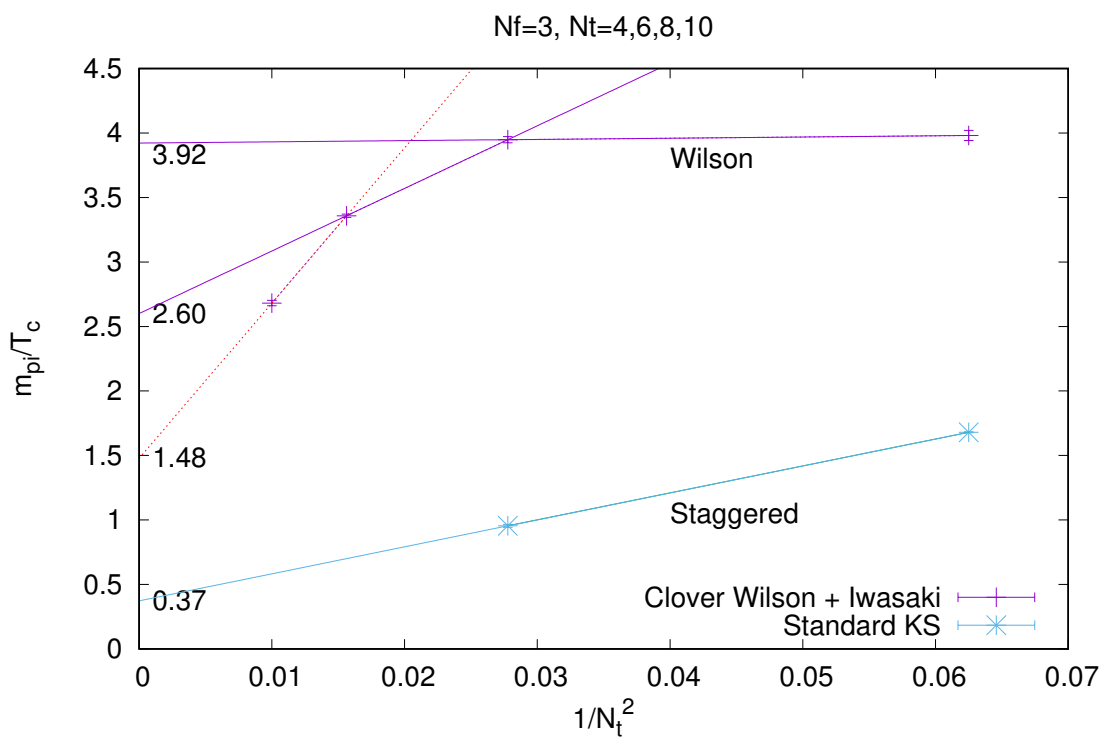

Figure 2: $N_{f}=3$ comparison of $m_{\pi}^{c} / T_{c}$ as a function of the lattice spacing $a^{2}$, between standard staggered fermions [8] and non-perturbatively improved Wilson fermions [5]. The $N_{t}=4$ and 6 Wilson fermion data would mistakenly suggest small lattice artifacts. The $N_{t}=10$ Wilson point was presented at the Lattice conference [11]. The numbers along the $y$-axis indicate the results of linear extrapolations in $a^{2}$.

down to arbitrarily small quark masses! In contrast, with Wilson fermions Ref. [5] finds a critical "pion" mass of about $300 \mathrm{MeV}$ after extrapolating from $N_{t}=6$ and 8.

Thus, one is led to mistrusting the staggered simulations for two reasons: they indicate a very small critical "pion" mass, consistent with zero; and they disagree strongly with Wilson fermion results, both at finite lattice spacing and after continuum extrapolation.

One plausible culprit for these two puzzles might be rooting. With staggered fermions, the Dirac determinant is raised to the power $3 / 4$ to mimic $N_{f}=3$ degenerate flavors. The danger of this procedure has been pointed out [14]: the consensus is that danger appears when the chiral limit is approached first, before the continuum limit is taken. This is potentially the case here, since the quark masses needed for criticality quickly approach zero as $N_{t}$ is increased.

To eliminate a possible issue with rooting, we have studied the case of $N_{f}=4$ degenerate flavors: no rooting is required, and the thermal transition in the chiral limit is expected to be first-order as for $N_{f}=3$. Actually, a naive counting of the degrees of freedom suggests that the first-order transition will be stronger for $N_{f}=4$ than for $N_{f}=3$, so that the critical "pion" mass will be heavier, thus reducing the computing cost of the simulations.

\section{Results: $N_{f}=4$}

As argued above, we have simulated standard staggered fermions with $N_{f}=4$ flavors and Wilson plaquette action, in order to bypass potential harmful effects of rooting and to keep computing costs down. The numerical simulations have been performed using a code running on GPUs [15]. 
For successive values of $N_{t}=4,6,8$ and 10, we have simulated lattices of spatial size $N_{s} \geq 2 N_{t}$, and determined the light bare quark mass $m_{q}^{c}$ for which the finite-temperature chiral transition is second-order. Following [3], the order of the phase transition was established by monitoring the Binder cumulant $B_{4}(\bar{\psi} \psi)$, where $B_{4}(X) \equiv \frac{\left\langle(\delta X)^{4}\right\rangle}{\left\langle(\delta X)^{2}\right\rangle^{2}}$ and $\delta X \equiv X-\langle X\rangle$. Near criticality, $B_{4}$ should be a function of the ratio $L_{S} / \xi$ of the spatial lattice size over the spatial correlation length, which diverges as $\left|m_{q}-m_{q}^{c}\right|^{-v}$. The critical value 1.604.. and the critical exponent $v \approx 0.63$ are known from the $3 d$ Ising universality class. Thus, one can expand $B_{4}\left(m_{q}\right)$ near $m_{q}^{c}$ as

$$
B_{4}\left(m_{q}\right)=1.604+c_{1}\left(m_{q}-m_{q}^{c}\right) N_{s}^{1 / v}+\mathscr{O}\left(\left(m_{q}-m_{q}^{c}\right)^{3}\right)
$$

An illustrative fit (including cubic terms) is shown in Fig. 3 (left) for $N_{t}=4$.

For a given value of $N_{t}$, this procedure determines the bare parameters $a m_{q}^{c}$ and $\beta$ required for criticality. A zero-temperature simulation is then performed, at these parameters, to determine the $T=0$ pion mass $\left(a m_{\pi}^{c}\right)\left(N_{t}\right)$. Finally, one obtains the physically meaningful ratio $m_{\pi}^{c} / T_{c}=N_{t} a m_{\pi}^{c}$, and repeats this procedure for successive values of $N_{t}$. These successive ratios are shown in Fig. 3 (right).

The zero-temperature simulations require large lattices $\left(N_{s}^{0}\right)^{3} \times N_{t}^{0}$, both spatially (to maintain $\left(m_{\pi} L_{s}\right) \gg 1$ ) and temporally (to achieve $T \approx 0$ ). To reduce the computing effort, we chose $N_{s}^{0} \geq 2 N_{t}$ and $N_{t}^{0} \geq 4 N_{t}$, i.e. we used a $20^{3} \times 40$ "zero-temperature" lattice in combination with a $20^{3} \times 10$ finite-temperature lattice. We are aware that our choice is only marginally satisfactory, and causes systematic errors in the extracted pion mass. However, Fig. 3 (right) shows variations of order $100 \%$ as $N_{t}$ is varied, which makes our systematic errors negligible in comparison.
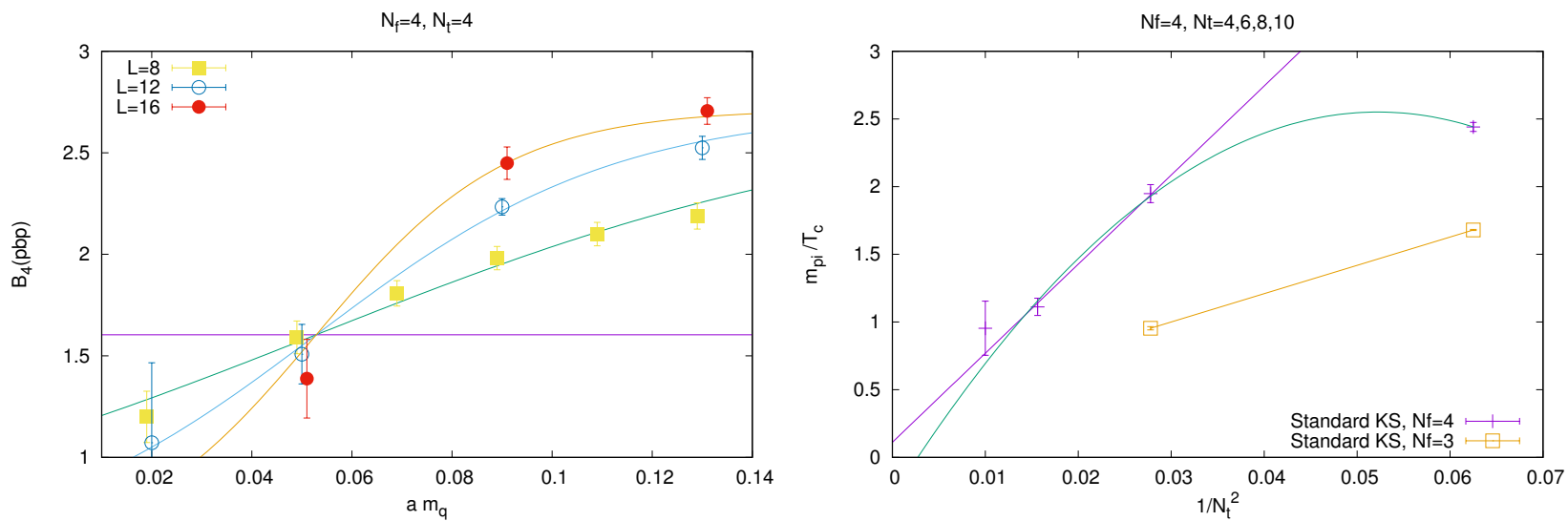

Figure 3: (left): finite-size scaling of the Binder cumulant $B_{4}(\bar{\psi} \psi)$ with the bare quark mass, here for $N_{t}=4$. (right): $N_{f}=4$ variation of $m_{\pi}^{c} / T_{c}$ as a function of the lattice spacing $a^{2}$, with linear and quadratic extrapolations. $N_{f}=3$ staggered results are shown for comparison: the critical "pion" mass is smaller, as expected.

\section{Discussion}

Our $N_{f}=4$ results Fig. 3 (right) are qualitatively similar to previous $N_{f}=3$ results: the dramatic reduction or $m_{\pi}^{c} / T_{c}$ as the lattice spacing is reduced is still present, and thus not related 
to rooting. Actually, a similar reduction is now visible in the Wilson data as well, with the new $N_{t}=10$ point [11] (see Fig. 2). Therefore, there is no reason to doubt the universality of the continuum limit: the Wilson and the staggered values should converge as $N_{t}$ is increased.

What is remarkable, however, is how slow this convergence is, even with a non-perturbatively improved action as in the Wilson case! Note that $N_{t}=10$, from which the continuum value of $m_{\pi}^{c} / T_{c}$ will probably differ by a factor 2 or more, corresponds to a lattice spacing $a \sim 0.13 \mathrm{fm}$. State-of-the-art thermodynamic studies use a maximum number of $N_{t}=16$ time-slices.

Let us speculate on the reason for such large cutoff effects. Taste-breaking could be the explanation: $N_{f}=4$ staggered fermions possess 16 "pions", but only one of them is really light, and the 15 others become degenerate with it in the continuum limit only. Thus, as the lattice spacing is reduced, the number of light pions effectively increases. But this should make the transition stronger, not weaker as observed. Moreover, the opposite occurs with Wilson fermions: there, the doublers become heavier toward the continuum limit. Nevertheless, Wilson and staggered fermions both lead to the transition becoming softer in the continuum limit. Thus, the explanation must reside elsewhere.

Perhaps cutting off all but the $N_{t}$ lowest Matsubara frequencies has larger than expected consequences. One simple exercise consists of calculating the pressure of a free massless boson on the lattice, and comparing it with the continuum Stefan-Boltzmann law [16]. An instructive figure can be found in Fig. 2 of [17]. It shows that the lattice pressure can easily differ from the continuum one by factors well beyond 10. The pressure deficit due to the Matsubara cutoff is less pronounced if the boson is more massive, so that the cutoff will extend the parameter regime of the confined phase, and push the critical "pion" mass upward.

If this guess is correct, then it is essential to reduce the temporal lattice spacing, not so much the spatial one. Lattice actions with anisotropic couplings would afford an economical approach to the continuum limit. For the measurement of $m_{\pi}^{c} / T_{c}$, the non-perturbative tuning of the (gauge and fermion) anisotropy coefficients is not needed, as long as the continuum limit is consistent. Alternatively, one could improve the action so as to approach the Stefan-Boltzmann law better, in the spirit of the p4-improved action [18].

Controlling the continuum extrapolation of the $N_{f}=4$ finite temperature transition will serve us for the $N_{f}=2+1$ case as well. There, unexpected results have been obtained for the upper-left corner of the Columbia plot: the thermal transition appears to be first-order - on a coarse, $N_{t}=4$ lattice [19]. Lattice corrections should also be carefully considered in the search for a conformal window in the number of flavors: it has been proposed to identify the lower edge of this window as the number of massless flavors for which the critical temperature of the chiral phase transition reaches zero [20] - there too, lattice corrections may well be very significant.

Finally, it is clear that the continuum value of $m_{\pi}^{c} / T_{c}$, for $N_{f}=4$ and even more so for $N_{f}=$ 3 , is going to be extremely small. At present, continuum extrapolations as in Fig. 3 (right) are compatible with a zero value. Could it actually be exactly zero, in contradiction with the predictions of $[1]$ ?

\section{Acknowledgments}

Numerical simulations have been performed on a GPU farm located at the INFN Computer 
Center in Pisa and on the QUONG cluster in Rome.

\section{References}

[1] R. D. Pisarski and F. Wilczek, Phys. Rev. D 29 (1984) 338.

[2] A. Butti, A. Pelissetto and E. Vicari, JHEP 0308 (2003) 029 doi:10.1088/1126-6708/2003/08/029 [hep-ph/0307036].

[3] P. de Forcrand and O. Philipsen, Nucl. Phys. B 673 (2003) 170 [hep-lat/0307020]; JHEP 0811 (2008) 012 [arXiv:0808.1096 [hep-lat]].

[4] C. Alexandrou, A. Borici, A. Feo, P. de Forcrand, A. Galli, F. Jegerlehner and T. Takaishi, Phys. Rev. D 60 (1999) 034504 [hep-lat/9811028]. H. Saito et al. [WHOT-QCD Collaboration], Phys. Rev. D 84 (2011) 054502 Erratum: [Phys. Rev. D 85 (2012) 079902] [arXiv:1106.0974 [hep-lat]]. M. Fromm, J. Langelage, S. Lottini and O. Philipsen, JHEP 1201 (2012) 042 [arXiv:1111.4953 [hep-lat]].

[5] X. Y. Jin, Y. Kuramashi, Y. Nakamura, S. Takeda and A. Ukawa, Phys. Rev. D 91 (2015) no.1, 014508 [arXiv:1411.7461 [hep-lat]].

[6] F. Karsch, E. Laermann and C. Schmidt, Phys. Lett. B 520 (2001) 41 [hep-lat/0107020].

[7] F. Karsch, C. R. Allton, S. Ejiri, S. J. Hands, O. Kaczmarek, E. Laermann and C. Schmidt, Nucl. Phys. Proc. Suppl. 129 (2004) 614 [hep-lat/0309116].

[8] P. de Forcrand, S. Kim and O. Philipsen, PoS LAT 2007 (2007) 178 [arXiv:0711.0262 [hep-lat]].

[9] H.-T. Ding, A. Bazavov, P. Hegde, F. Karsch, S. Mukherjee and P. Petreczky, PoS LATTICE 2011 (2011) 191 [arXiv:1111.0185 [hep-lat]].

[10] L. Varnhorst, PoS LATTICE 2014 (2015) 193.

[11] S. Takeda, X. Y. Jin, Y. Kuramashi, Y. Nakamura and A. Ukawa, arXiv:1612.05371 [hep-lat].

[12] A. Bazavov et al., Phys. Rev. D 85 (2012) 054503 [arXiv:1111.1710 [hep-lat]].

[13] G. Endrodi, Z. Fodor, S. D. Katz and K. K. Szabo, PoS LAT 2007 (2007) 182 [arXiv:0710.0998 [hep-lat]].

[14] M. Creutz, PoS LAT 2007 (2007) 007 [arXiv:0708.1295 [hep-lat]]. C. Bernard, M. Golterman, Y. Shamir and S. R. Sharpe, Phys. Rev. D 77 (2008) 114504 [arXiv:0711.0696 [hep-lat]].

[15] C. Bonati, G. Cossu, M. D’Elia and P. Incardona, Comput. Phys. Commun. 183, 853 (2012) [arXiv:1106.5673 [hep-lat]].

[16] J. Engels, F. Karsch and H. Satz, Nucl. Phys. B 205 (1982) 239.

[17] P. de Forcrand, P. Romatschke, W. Unger and H. Vairinhos, PoS LATTICE 2016 (2017) 086.

[18] U. M. Heller, F. Karsch and B. Sturm, Phys. Rev. D 60 (1999) 114502 [hep-lat/9901010].

[19] C. Bonati, P. de Forcrand, M. D’Elia, O. Philipsen and F. Sanfilippo, Phys. Rev. D 90 (2014) no.7, 074030 [arXiv:1408.5086 [hep-lat]].

[20] K. Miura, M. P. Lombardo and E. Pallante, Phys. Lett. B 710 (2012) 676 [arXiv:1110.3152 [hep-lat]]. 